\title{
The WELL Detector
}

\author{
R. Bellazzini ${ }^{\mathrm{a}, \mathrm{b}} \mathrm{M} \mathrm{Bozzo}^{\mathrm{c}}$ A. Brez ${ }^{\mathrm{a}}$ G. Gariano ${ }^{\mathrm{a}}$ L. Latronico $^{\text {a }}$ \\ N. Lumb ${ }^{\text {a }}$ A. Papanestis ${ }^{\text {a }}$ G. Spandre ${ }^{\text {a }}$ M.M. Massai ${ }^{a}$ R. Raffo ${ }^{a}$ \\ M.A. Spezziga ${ }^{a}$ \\ ${ }^{\mathrm{a}}$ INFN-Pisa and University of Pisa, Pisa, Italy \\ ${ }^{\mathrm{b}}$ CERN, CH-1211 Geneva 23, Switzerland \\ ${ }^{\mathrm{c}}$ INFN-Genova and University of Genova, Italy
}

\begin{abstract}
We introduce the WELL detector, a new type of position-sensitive gas proportional counter produced using advanced printed circuit board (PCB) technology. The WELL is based on a thin kapton foil, copperclad on both sides. Charge amplifying micro-wells are etched into the first metal and kapton layers. These end on a micro-strip pattern which is defined on the second metal plane. The array of micro-strips is used for read-out to obtain 1-D positional information. First results from our systematic assessment of this device are reported.
\end{abstract}

\section{Introduction}

The trend in the design and construction of modern gas detectors is the use of sophisticated patterning technology to define micro-electrodes for charge collection and amplification. Recently it has been shown that it is possible to use advanced printed circuit board (PCB) techniques to fabricate the amplifying structures. This technology has been employed as an alternative to the more delicate and expensive micro-electronics based procedures used in the production of detectors such as the Micro-Strip Gas Counter (MSGC) [1,2] or the Micro-Gap Counter (MGC) [3].

Examples of detectors built using advanced PCB technology are the Gas Electron Multiplier (GEM) [4] and the recently introduced Micro-Groove Detector (MGD) [5]. Work to date with these devices suggests that although the size of the smallest electrode structure is almost ten times greater than can be achieved with micro-electronics techniques, their performance in terms of gain, rate capability, energy resolution, etc., is comparable with that of the MSGC and related detectors. The MGD in particular retains many of the desirable characteristics of the MSGC (including the electrical and mechanical simplicity of a single gas gap) while offering the possibility of true 2-D read-out and significant advantages in the areas of cost and robustness. 


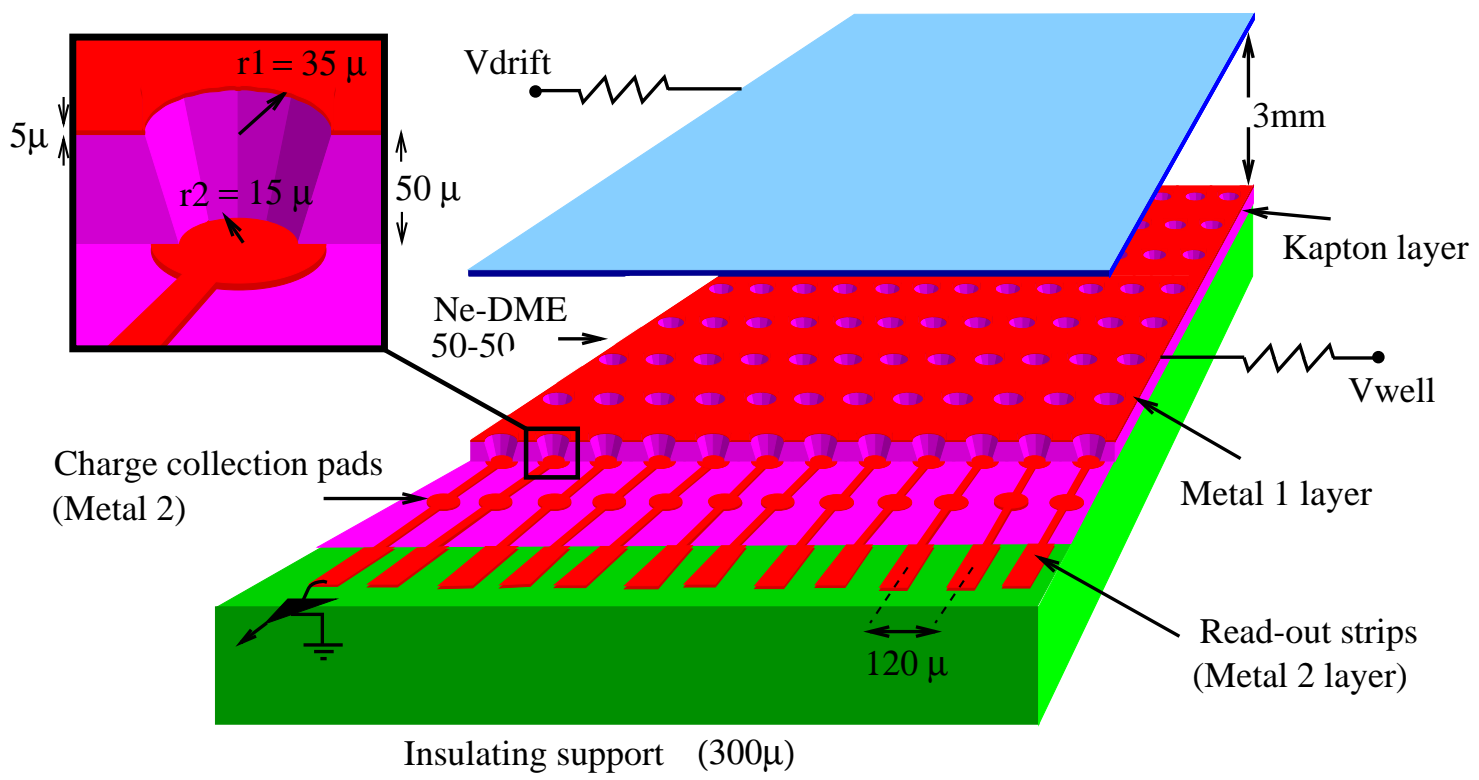

Fig. 1. Schematic diagram of a WELL detector.

We have developed a 1-D version of the MGD called the WELL detector, which further demonstrates the flexibility of the PCB approach. As with the MGD, the starting-point for construction of a WELL detector is a thin $(50 \mu \mathrm{m})$ kapton foil, coated with gold-plated copper on both surfaces (we refer to these coatings as metal-1 and metal-2). In the MGD, micro-grooves are etched in the metal-1 and kapton layers. The parallel strips remaining on the metal-1 plane are used to extract position information. A second micro-strip pattern is defined on the metal-2 layer at an angle to the metal-1 strips, providing a second coordinate. The kapton foil is glued to a vetronite or carbon fibre support and separated from a drift window by a single $3 \mathrm{~mm}$ spacer. Application of high voltage between the two metal layers in a suitable gas environment creates an electric field in the micro-grooves which is intense enough to produce charge amplification.

In the WELL detector, the grooves are replaced by charge-amplifying micro-wells (Fig. 1). Because of their cylindrical symmetry, field lines are focused into the micro-wells from all possible directions and not only from the sides as in the MGD, see Fig. 2. As a result, the electric field and thus the gas gain are expected to be significantly higher for the WELL detector than for the MGD, for the same applied voltage. In its simplest implementation (as reported in this paper) this design gives only 1-D position information. The possibility of a 2-D WELL detector exists and will be the subject of future investigations.

\section{Details of test detector}

The PCB technology used to fabricate the WELL is the same as that employed for construction of the MGD and can be considered a modification of the techniques 


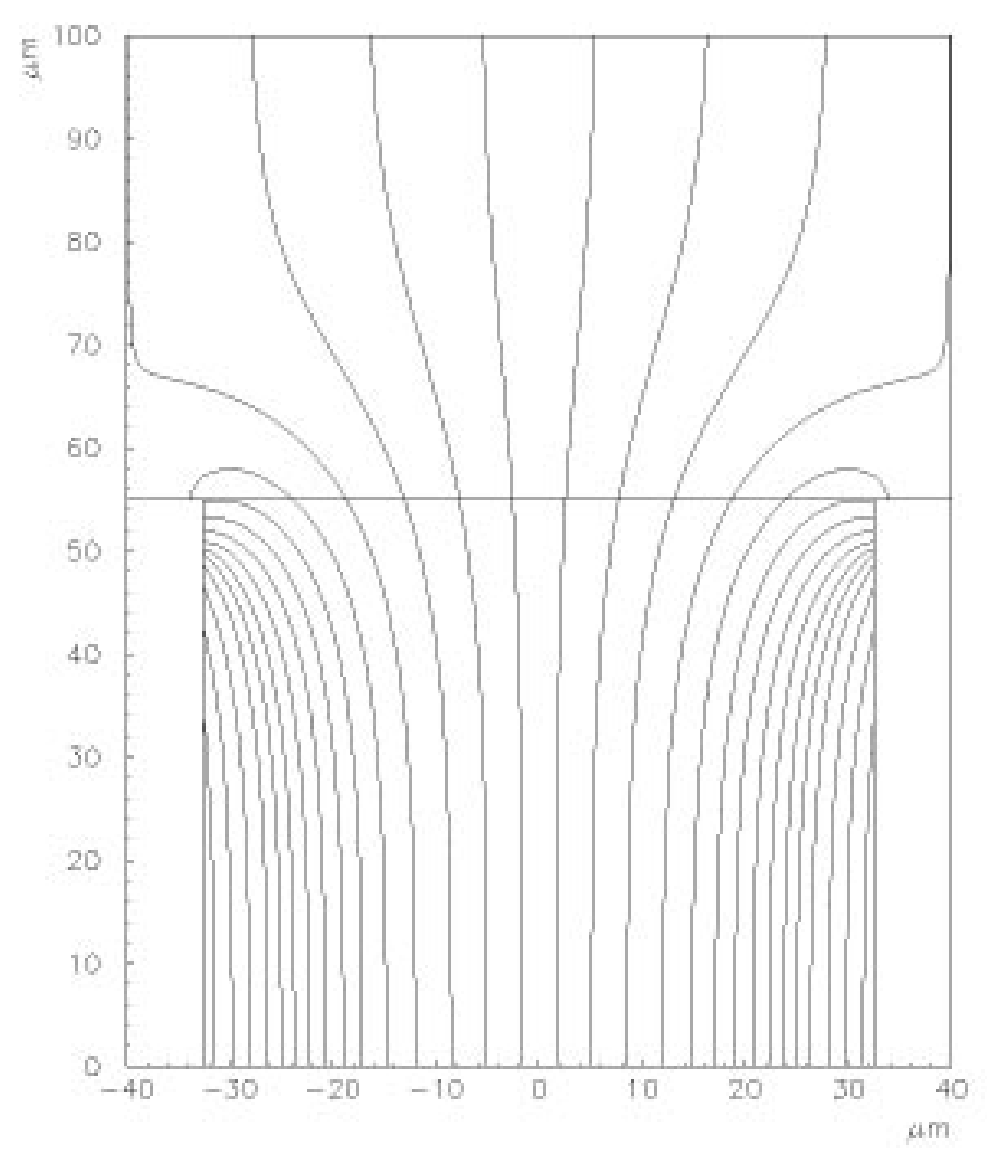

Fig. 2. Electric field map of one cell of a WELL detector.

used to build the GEM [6]. The main difference between a GEM and the WELL is that the GEM alone acts only as an amplifying stage and gives no positional information. To obtain such information, an extra plane of collection electrodes must be added to the detector, separated from the GEM by a distance of typically $3 \mathrm{~mm}$ (in addition to the $3 \mathrm{~mm}$ gas gap between the GEM and the drift window). Early work [4] used an MSGC for this purpose, as it was thought that the gain of the GEM was not sufficient to produce detectable signals. Since then it has been discovered that the GEM itself can reach gas gains of several thousand [7,8], allowing the use of a simple array of copper collection strips for read-out to obtain 1-D positional information.

It is clear that there is a big advantage in placing the read-out strips directly onto the kapton foil, as in the case of the WELL, eliminating the mechanical construction complications of an external, separate plane of electrodes. Furthermore, it is desirable to keep the gas gap as thin as possible in order to work with a manageable high voltage on the drift. This requirement derives from the fact that the drift field of a gaseous detector operated in the high magnetic fields of modern particle physics experiments must be high enough to maintain a relatively small Lorenz angle. Both the MGD and the WELL detector appear to overcome these problems. These devices potentially have the additional benefit of improved response unifor- 

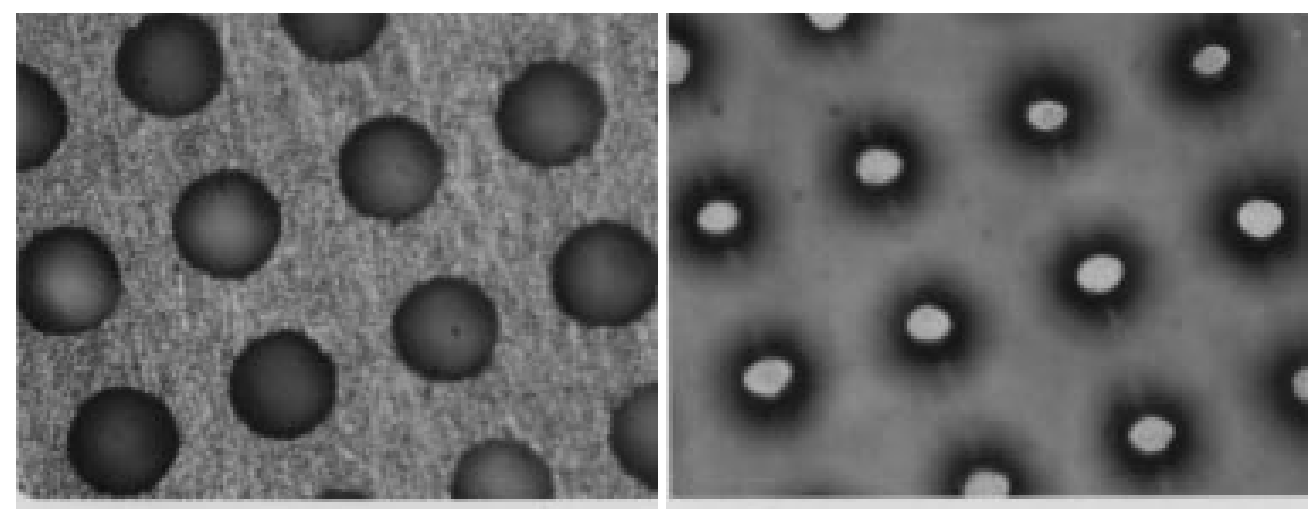

Fig. 3. Microscope photographs of a WELL detector, with the focus at the top (left image) and bottom (right image) of the wells.

mity, which in a GEM, for example, is strongly dependent upon the flatness of the metallised kapton layer with respect to the collection plane [9].

The device used for the tests reported in this paper was based upon a $3 \times 3 \mathrm{~cm}$ square of copper-clad kapton. A square array of circular wells was etched in the metal- 1 and kapton layers (thicknesses 5 and 50 microns respectively), the distance between hole centres being $120 \mu \mathrm{m}$. The hole diameter was $68 \mu \mathrm{m}$ in the plane of the metal and approximately $30 \mu \mathrm{m}$ at the bottom of the kapton wells (the conical shape of the wells is a consequence of the etching process). Fig. 3 shows microscope photographs taken from above the wells, with the focus at the top (left image) and bottom (right image) of the wells. In the metal-2 layer, circular charge collection pads, diameter $70 \mu \mathrm{m}$, were etched concentric to the wells. Strips of width $50 \mu \mathrm{m}$, also in the metal-2 layer, were used to interconnect the collection pads. These read-out strips were terminated in bonding pads for connection to the amplifying electronics.

The prepregging technique [10] was used to bond the kapton foil to a $300 \mu \mathrm{m}$ thick vetronite support. Finally, an aluminised mylar drift window was added, separated from the detection layer by a $3 \mathrm{~mm}$ spacer. The resulting detector (see Fig. 4) is, in terms of assembly procedure, identical to the MSGC substrate/spacer/window system. However the WELL construction materials make it extremely resistant to mechanical shock, overcoming the problems of fragility associated with chambers in which the electrodes are deposited on a thin glass substrate. After filling the gas envelope with a suitable gas mixture (e.g. Neon-DME, 50-50), voltages could be applied to the drift plane $\left(\mathrm{V}_{d}\right)$ and the upper (metal-1) WELL layer $\left(\mathrm{V}_{w}\right)$. The lower WELL electrodes were grounded via the pre-amplifiers. 


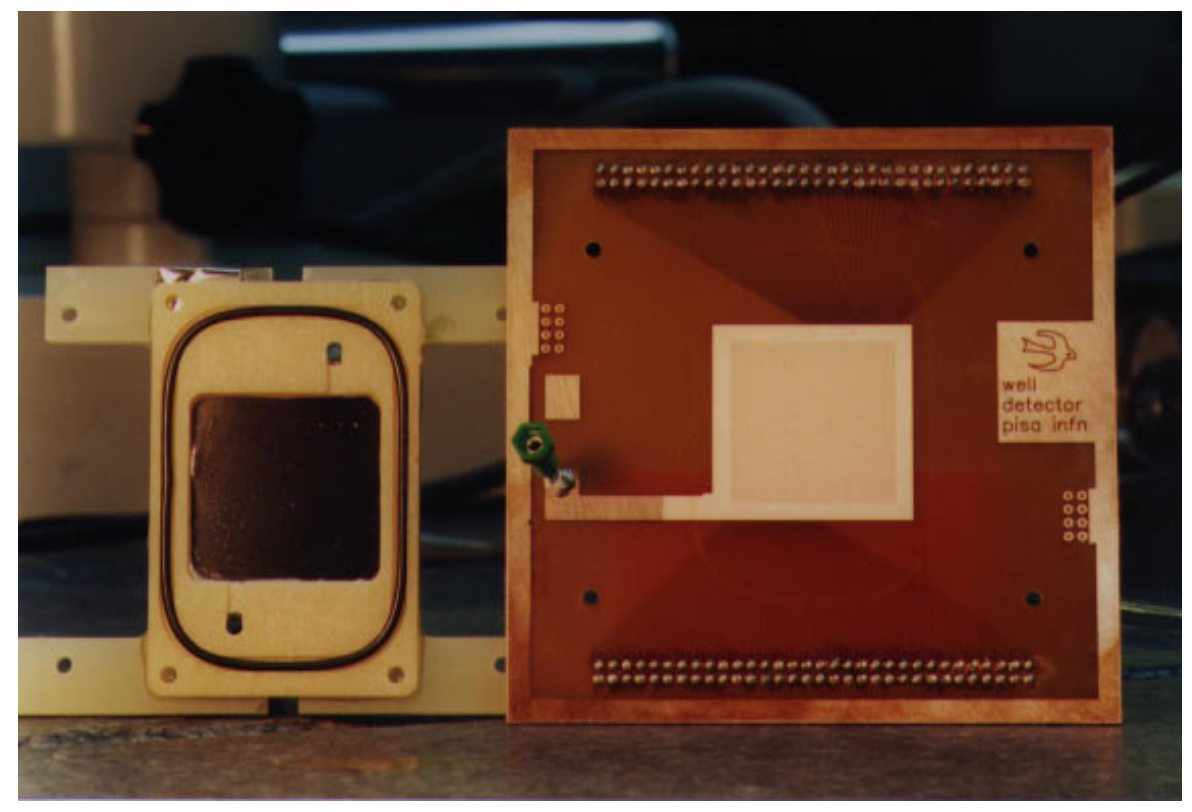

Fig. 4. A WELL detector just prior to final assembly, showing the drift window (left) and the copper-coated kapton foil glued to its vetronite support. The completed detector is mechanically very robust.

\section{Results and interpretation}

\subsection{Charge collection efficiency}

Some of the field lines in the drift region of a WELL detector are expected to terminate on the metal-1 layer. Primary electrons following these lines are not multiplied in the wells and therefore do not produce a detectable signal. The primary charge collection efficiency has been found to be dependent upon the strength of the drift field, see Fig. 5. We are now building a new version of the detector with the wells at a pitch of $90 \mu \mathrm{m}$ instead if $120 \mu \mathrm{m}$ and packed in a hexagonal array rather than a square one. From simulation studies of this configuration we expect to significantly improve the collection efficiency at very high drift fields. An assessment of the absolute collection efficiency has not yet been made; the maximum relative efficiency was obtained with a drift field strength of approximately $4200 \mathrm{~V} / \mathrm{cm}\left(\mathrm{V}_{d}=1700 \mathrm{~V}\right.$, $\mathrm{V}_{w}=450 \mathrm{~V}, 3 \mathrm{~mm}$ gas gap).

\subsection{Gas gain}

The gas gain of the detector was determined as a function of $\mathrm{V}_{w}$, for various different gas mixtures, see Fig. 6. At each measurement point, $\mathrm{V}_{d}$ was adjusted to maintain good collection efficiency. The detector was irradiated with $5.4 \mathrm{keV} \mathrm{Cr}$ $\mathrm{X}$-rays and the signal current $\mathrm{I}_{s}$ from the read-out strips was measured using a 


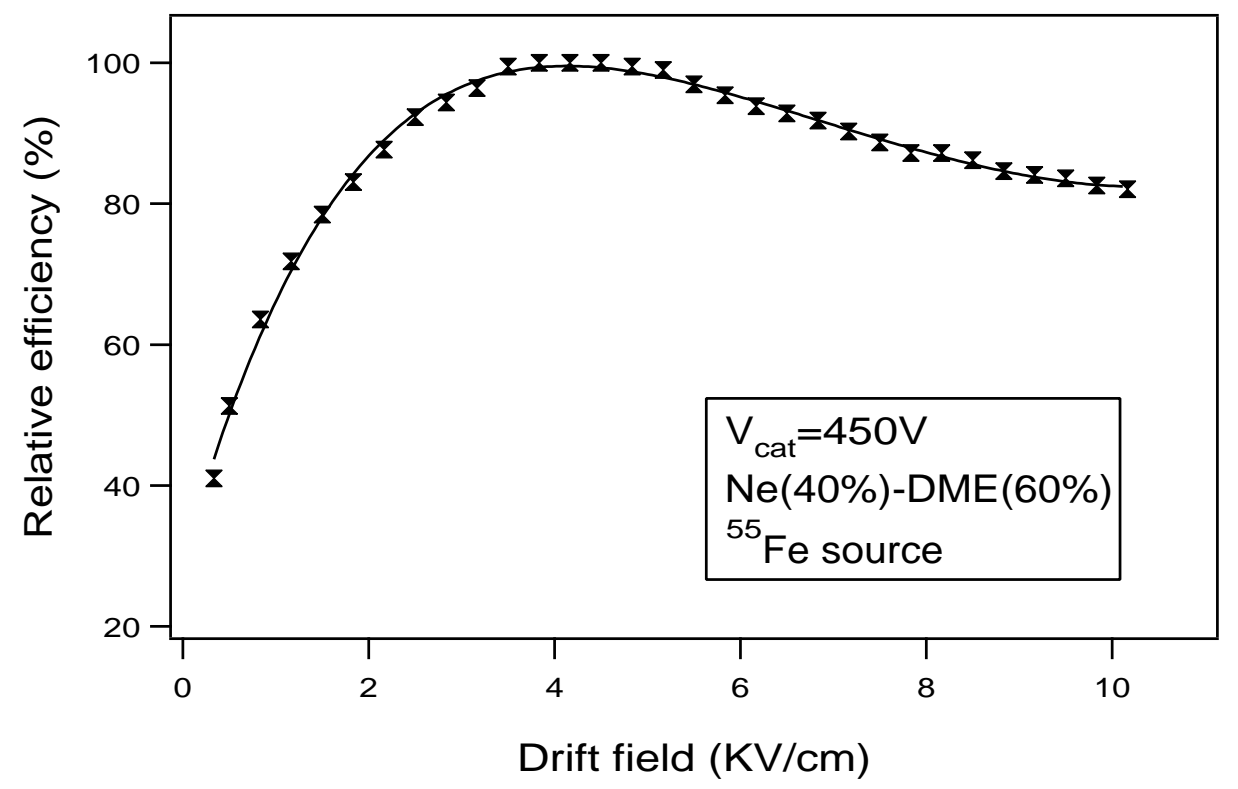

Fig. 5. Relative charge collection efficiency as a function of drift field strength.

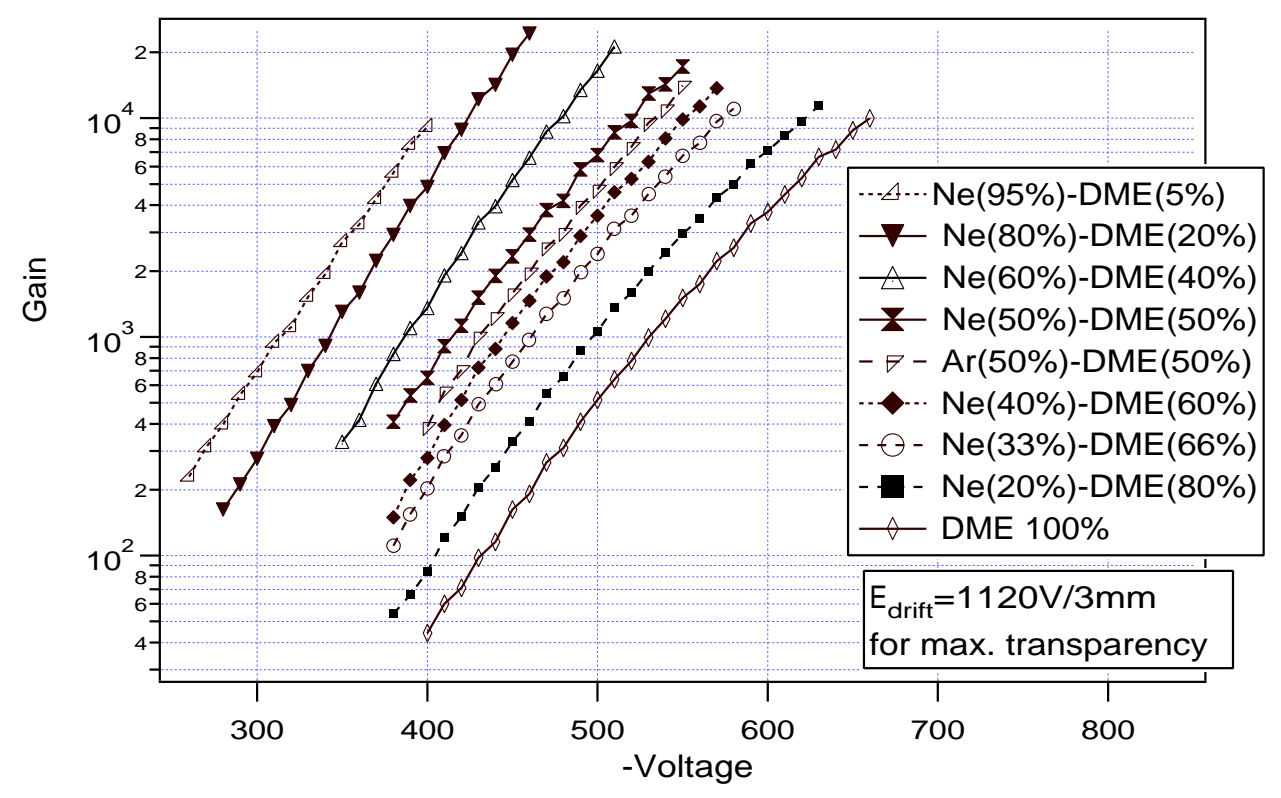

Fig. 6. Gas gain of the WELL detector for various gas mixtures.

pico-ammeter. Knowing the rate of the absorbed X-rays, and the average number of electrons produced in a primary ionisation, the gain could be calculated for each voltage $\left(\mathrm{I}_{s}=\right.$ Rate $*$ Gain $\left.* \mathrm{Q}_{\text {primary }}\right)$. The plot shows that gains above 10000 may easily be reached with this type of detector. For a given voltage on the upper WELL electrode, the gain may be enhanced by increasing the proportion of Neon in the gas. It was also noticed that the maximum achievable gain (after which the signal current became unstable) increases with Neon concentration, see Fig. 7. As expected, for the same voltage the gas gain of a WELL detector is higher by a factor 2 than that of an MGD (see [5]). This is because of the more symmetrical nature of 


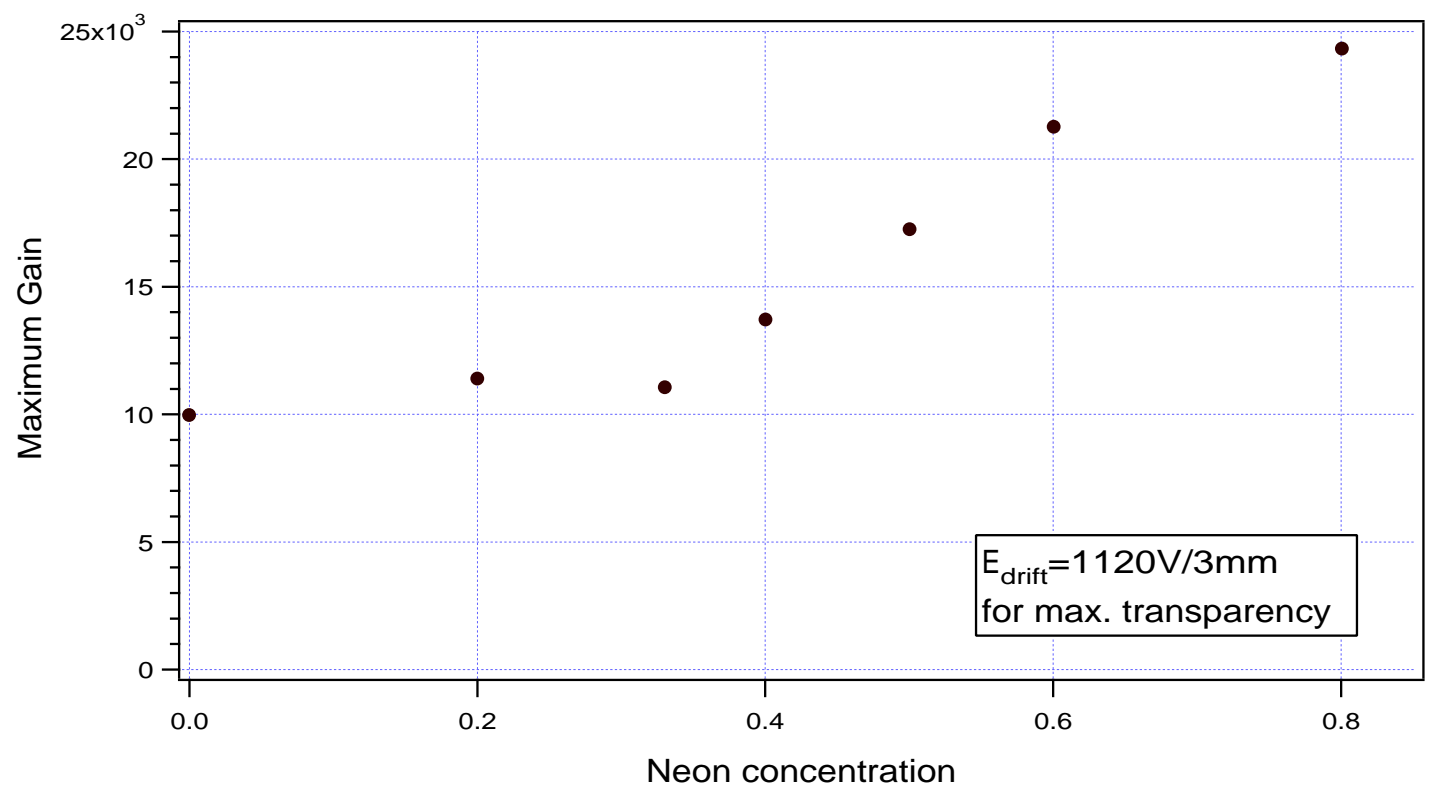

Fig. 7. Maximum achievable gain as a function of Neon concentration.

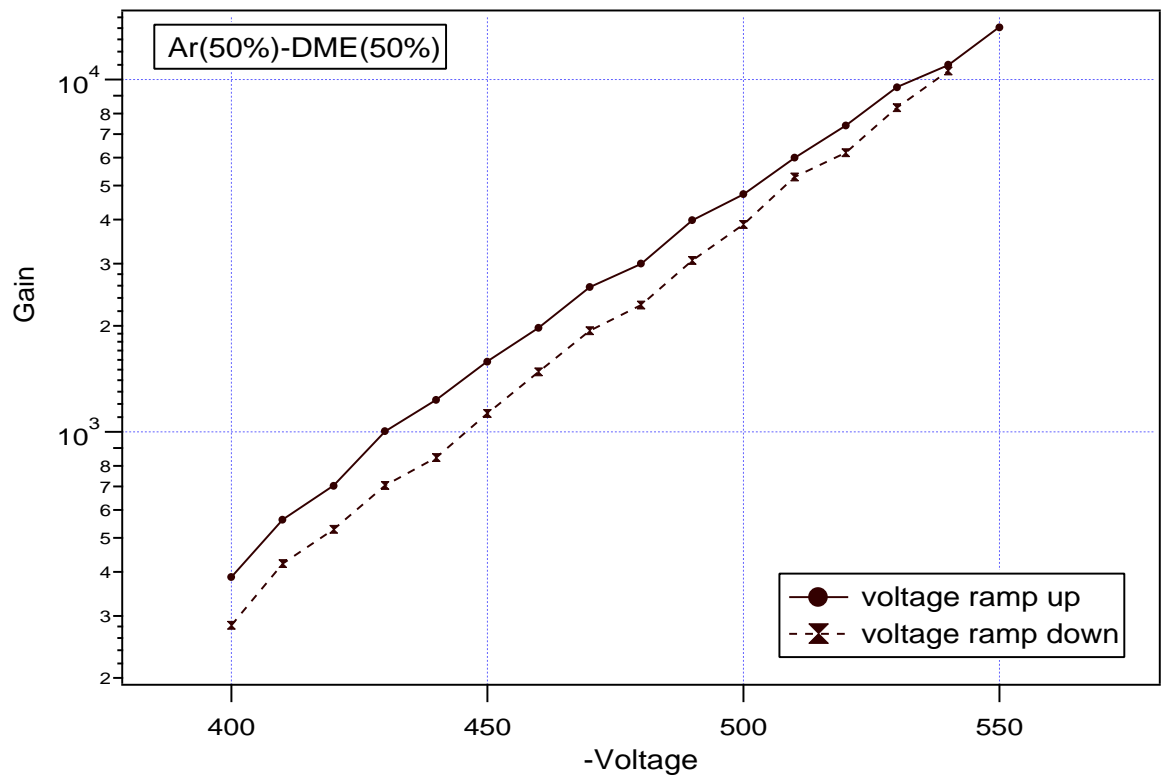

Fig. 8. Gain variation with voltage for Ar-DME (50-50), showing hysteresis effect.

the field configuration in the WELL.

The gain of the chamber was also checked using an Argon-DME, 50-50, gas mixture. The gain was measured as $\mathrm{V}_{w}$ was increased from $400 \mathrm{~V}$ to $550 \mathrm{~V}$ and also as the voltage was decreased from $550 \mathrm{~V}$ to $400 \mathrm{~V}$. A clear hysteresis effect was observed, see Fig. 8. This phenomenon is believed to be caused by polarisation within the kapton layer, an effect analogously observed in MSGCs (see section 3.4). 


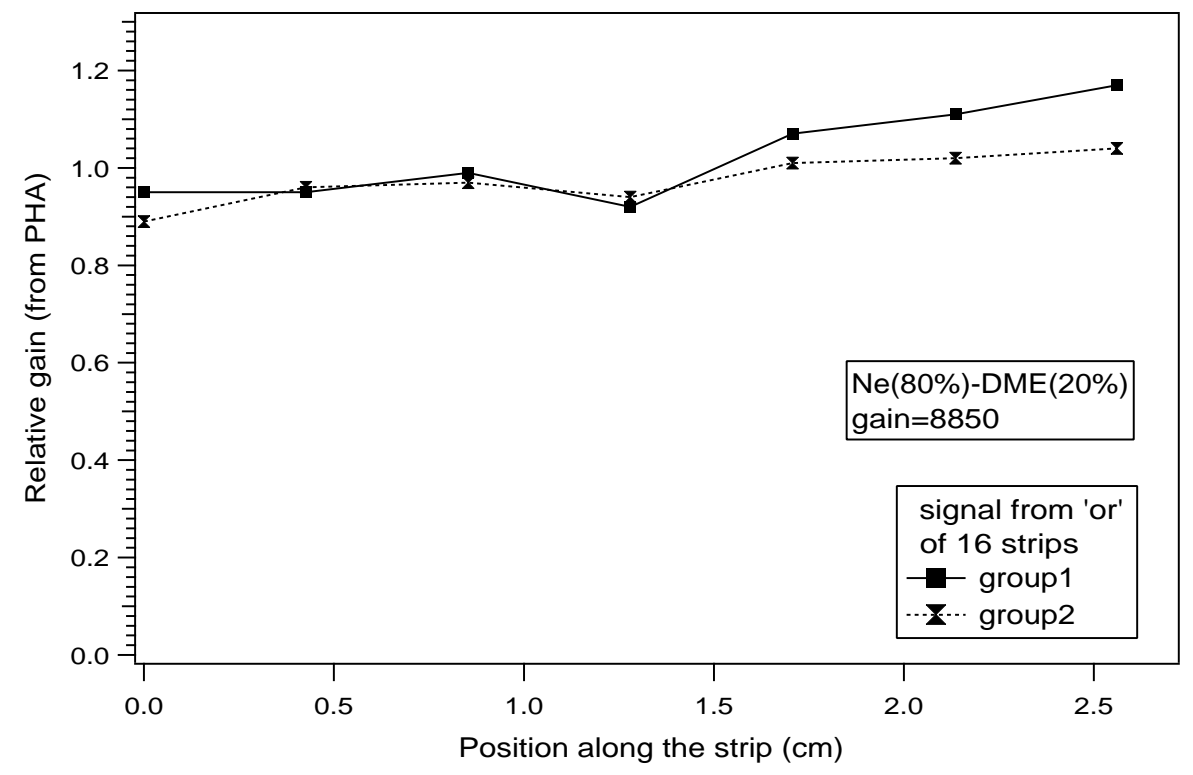

Fig. 9. WELL detector gain uniformity.

\subsection{Gain uniformity}

The dependence of gain on position of irradiation was studied. The signals from a group of 16 strips near the centre of the chamber were combined and the position of the peak in the pulse height spectrum corresponding to the $5.4 \mathrm{keV}$ photons was determined using a pulse height analyser (PHA). The relative gain (peak position/average peak position) at seven different points along the strips is plotted in Fig. 9. Results for a second, adjacent group of 16 strips are also shown. It can be seen that the gain was uniform over the fourteen different measurement positions within $15 \%$.

\subsection{Short-term stability}

The short-term gain stability of the detector was investigated by measuring the signal current from all of the strips for a period of five minutes after application of the radiation source, see Fig. 10. The test was performed at three different gain values, using a 50-50 mix of Neon-DME. At high gains, the signal current was observed to fall from an initially higher value, stabilising in less than a minute. At normal operating gains (around 2000), the effect is less pronounced and there is a small increase in gain. Such short-term instabilities are commonly observed for MSGCs and are attributed to polarisation effects in the substrate between the anodes and cathodes [11]. It seems logical to assume that a similar charging effect takes place in the kapton layer of a WELL detector, producing the phenomena described above. 


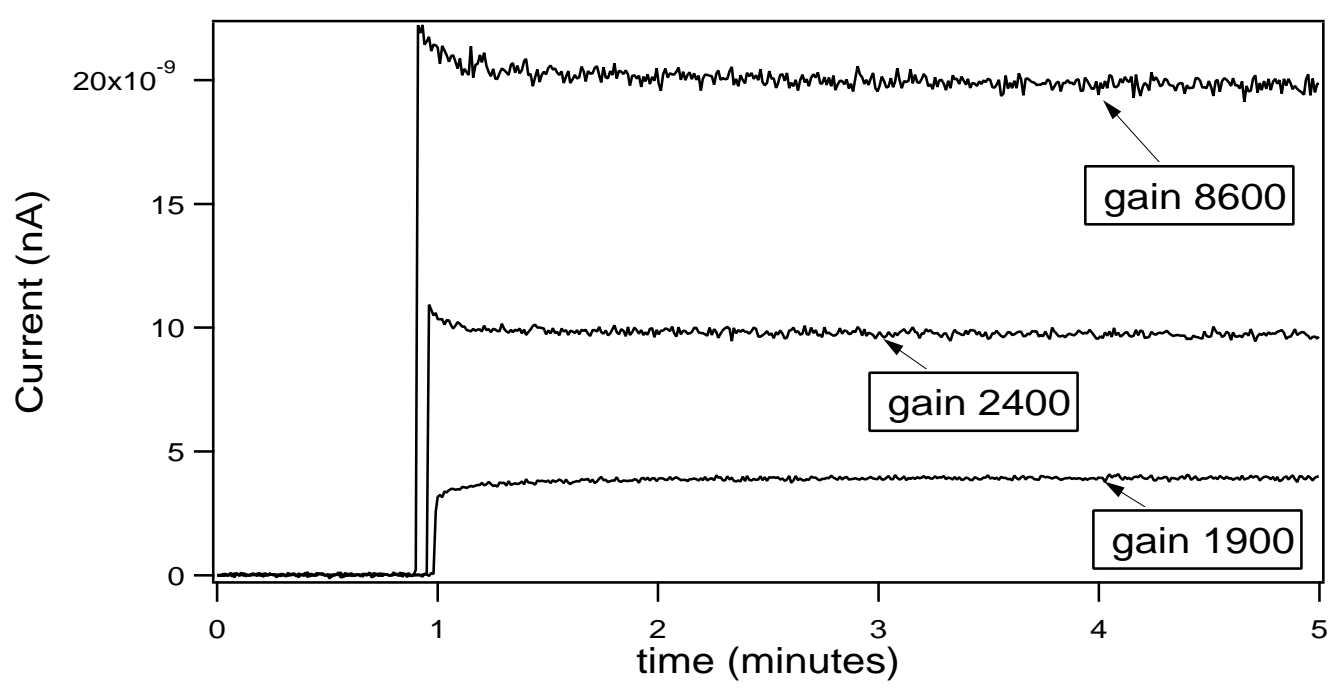

Fig. 10. Short-term gain stability.

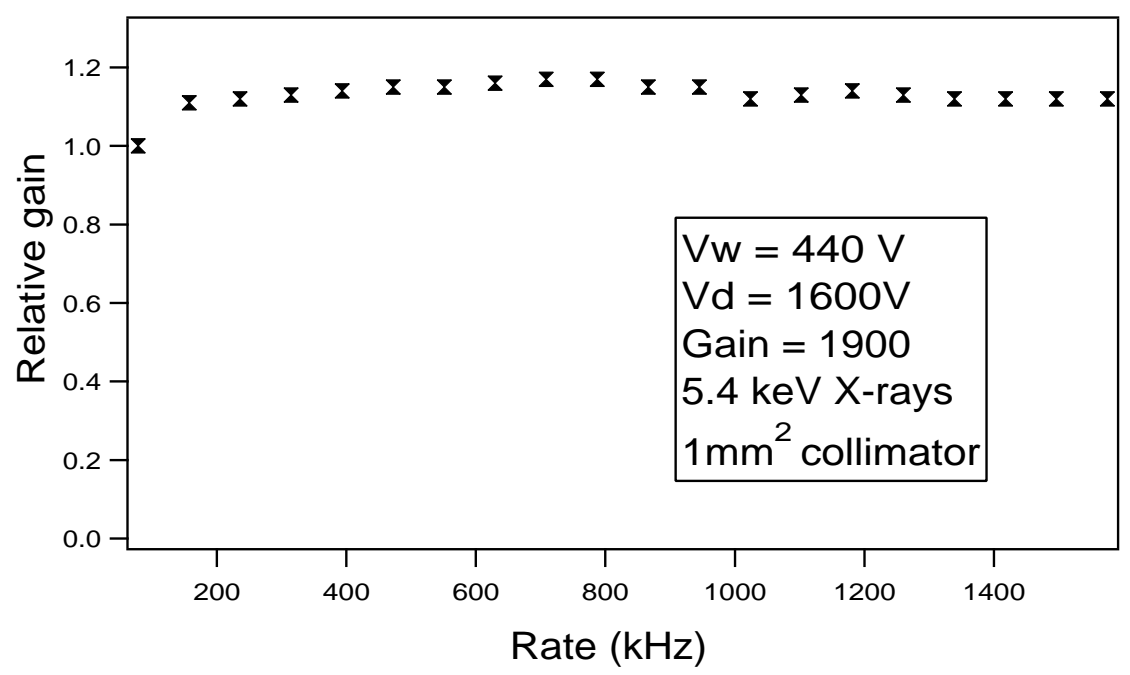

Fig. 11. Detector gain as a function of rate for $5.4 \mathrm{keV} \mathrm{X-rays.}$

\subsection{Rate capability}

The rate capability of the WELL detector was checked by irradiating the detector with $5.4 \mathrm{keV}$ photons from an X-ray tube (Cr target), for absorbed rates of up to $1.6 \times 10^{6}$ photons $/ \mathrm{mm}^{2} / \mathrm{s}$. The gain of the chamber was set to 1900 and the photon beam was collimated to a $1 \mathrm{~mm}^{2}$ spot. The results of this test are displayed in Fig. 11. The plot shows that no detectable drop in gain was observed over the full range of beam intensities. 

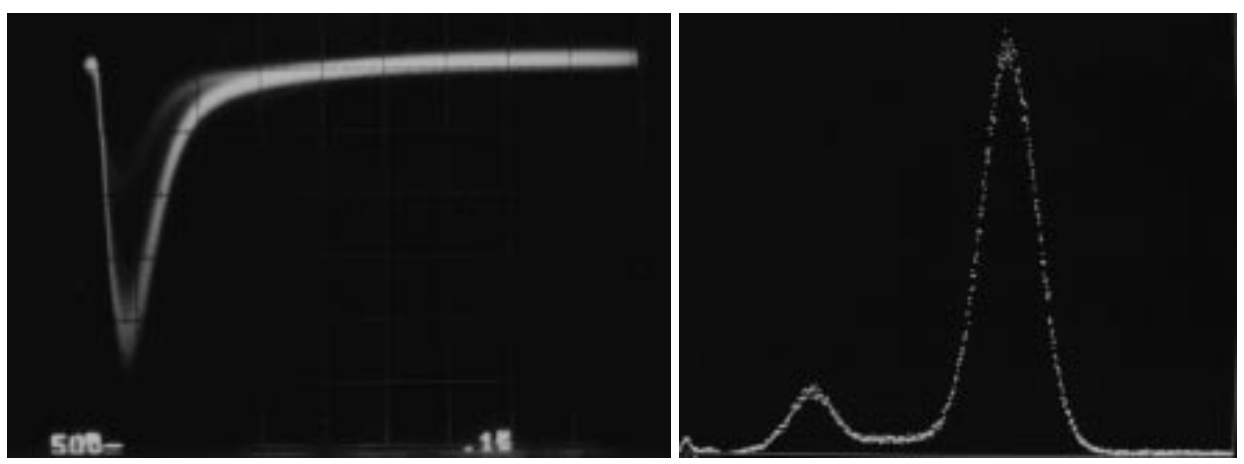

Fig. 12. Signal and pulse height spectrum from an OR of 6 strips, for Ar-DME (50-50). The argon escape peak is clearly resolved.

\subsection{Energy resolution}

The detector voltages were adjusted to the normal working values $\left(\mathrm{V}_{w}=430 \mathrm{~V}\right.$, $\mathrm{V}_{d}=1500 \mathrm{~V}$ ) to give a drift field of $3600 \mathrm{~V} / \mathrm{cm}$ for good charge collection efficiency and a gain of approximately 1000 in the Ar-DME (50-50) gas mixture used. The detector was irradiated with $5.4 \mathrm{keV}$ X-rays and the signals produced from an 'OR' of 6 strips (Fig. 12, left trace) were processed using the PHA, Fig. 12 (right trace). The signals from the principal photon energy at $5.4 \mathrm{keV}$ and from the argon 'escape' peak are clearly resolved in both the oscilloscope trace and the PHA spectrum. From the main peak the energy resolution was calculated to be $19 \%$ FWHM.

\subsection{Signal shape and ballistic deficit}

The signal from a WELL detector is compared with that from an MGC in Fig. 13 (upper and lower traces respectively). The rise and fall times of the pulse from the WELL are clearly longer than those of the MGC signal, implying slower charge collection.

If the collection time for charge at the electrodes of a detector is longer than the shaping time of the electronics used to amplify the signal, only a fraction of the full avalanche charge will be observed in the signal. This fraction, sometimes referred to as the ballistic deficit, was measured for the WELL detector using an amplifier with $20 \mathrm{~ns}$ integration and differentiation time constants. The electronics channel used was first of all calibrated by using a precision capacitor to inject a known amount of charge into the pre-amplifier. The height of the corresponding output pulse was measured and the calibration factor established to be 580 electrons $/ \mathrm{mV}$. This factor could then be used to estimate the charge, $\mathrm{Q}_{p h}$, corresponding to a signal of any given amplitude from the detector.

The WELL detector was operated under the standard working conditions with an Ar-DME (50-50) gas mixture. The pulse height and signal current were measured 


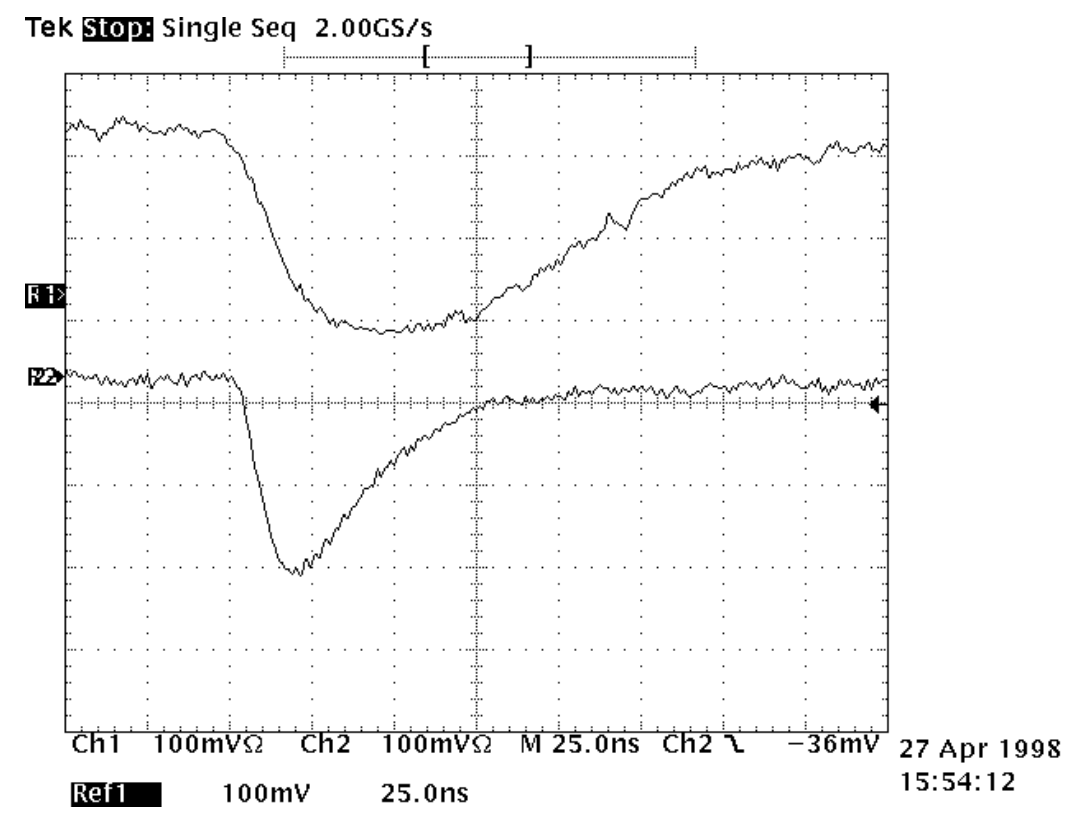

Fig. 13. Signals from a WELL detector (upper trace) and from an MGC (lower trace).

when the chamber was irradiated by $5.4 \mathrm{keV}$ photons at an absorbed rate of $9.6 \mathrm{kHz}$. The full avalanche charge could then be calculated $\left(\mathrm{Q}_{\text {full }}=\mathrm{I}_{s} /\right.$ Rate $)$ and the ballistic deficit $\left(\mathrm{Q}_{p h} / \mathrm{Q}_{\text {full }}\right)$ was found to be $55 \%$. This value, which is lower than the values measured for MSGCs and MGCs (67\% and $90 \%$ respectively) confirms that charge collection in the WELL detector is relatively slow.

\subsection{Response to heavily ionising particles (HIPs)}

A chamber operated at a gas gain of 2000 has been exposed to alpha particles at a rate of $2.5 \mathrm{kHz}$ over $1 \mathrm{~cm}^{2}$ of the detector area. A dedicated system [12] consisting of a pico-ammeter connected to a computer was used to monitor the frequency of any possible current spikes. Such instabilities are known to be associated with micro-discharges which may be induced by HIPs. The anodes of the WELL were short-circuited and connected in series with the pico-ammeter (Keithley 487, integration time $20 \mathrm{~ms}$ ), which fills a buffer with 512 readings and determines the maximum and minimum values. The pico-ammeter was monitored using the Labview data acquisition system (version 3.1), reading out and displaying the maximum current value of the buffer. If the reading was over threshold (set at $60 \mathrm{nA}$ ), the program counted one micro-discharge and began a further 512 acquisitions; if not, another acquisition series was started without the counter being incremented. The sequence was repeated until the required number of readings had been made. Reading only the maximum current value of the buffer reduces the dead-time to only $20 \%$. There were zero events over the threshold after $10^{8}$ alphas had been counted (see Fig. 14), corresponding to more than one year of LHC operation at full luminosity [13]. The chamber was opened at this point in the test and given 


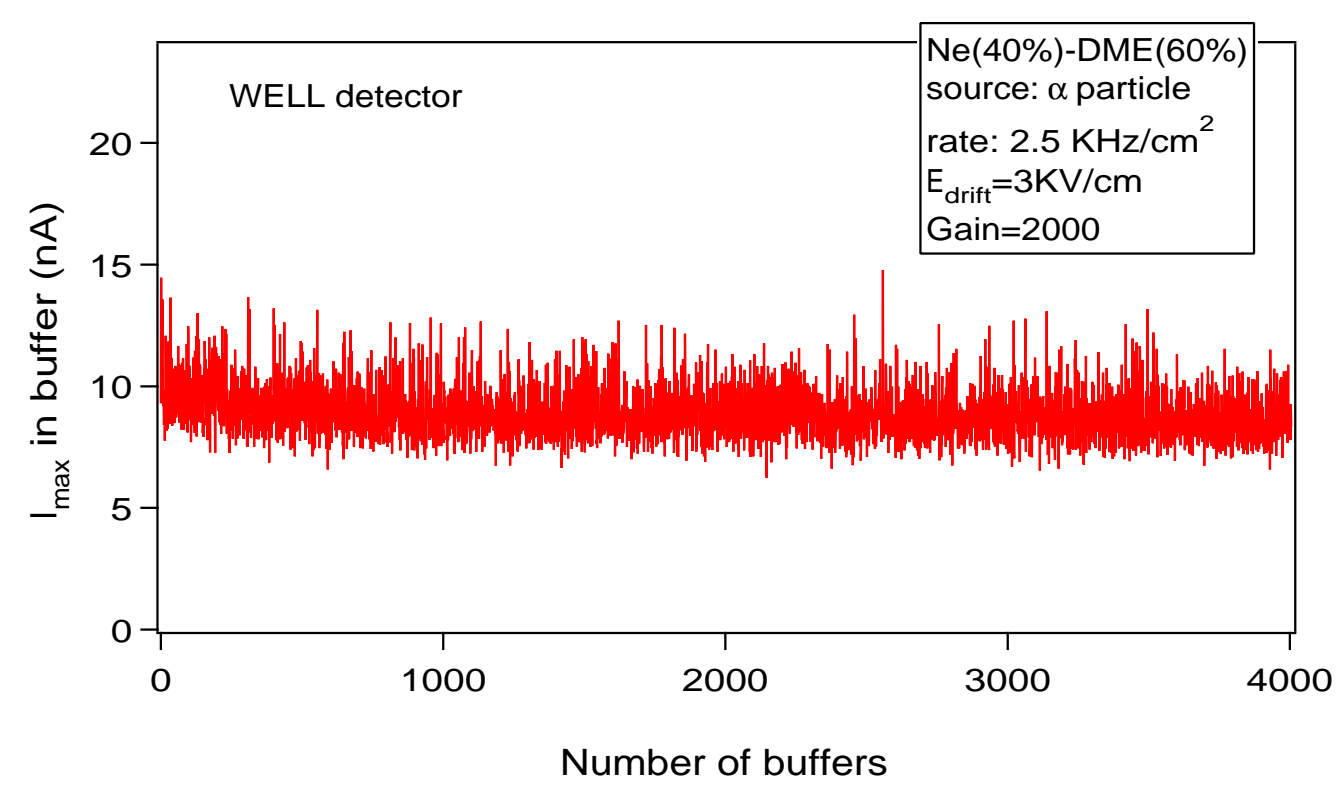

Fig. 14. Stability while exposed to HIPs: maximum signal current as a function of acquisition buffer number.

a full optical inspection under a microscope. No evidence of any damage to the detector was observed. Behaviour at higher gains is still to be investigated.

\section{Conclusions}

We have designed and tested a new type of position-sensitive gaseous proportional detector, which we call the WELL, based on advanced printed circuit board technology. Our preliminary investigations show that the device appears to have all of the desirable properties (large, uniform and stable gain; high rate capability; small cell size giving high granularity; good energy resolution) expected from a modern detector. Its performance competes well with that of other comparable devices (MSGCs, MGCs, etc.), apart from the fact that charge collection is somewhat slower. Nevertheless, the WELL still has sufficient speed for most of the applications that we can envisage. The fact that PCB techniques are used to fabricate the WELL, in contrast to the more delicate micro-electronics technology used for many of the detectors in this class, promises significant advantages in terms of mechanical and electrical robustness and cost.

We are now building WELL detectors with optimised geometry for improved collection efficiency at very high drift fields and with 2-D read-out. Results from the testing of these further developments will be reported soon.

\section{Acknowledgement}

We would like to acknowledge the assistance of A. Gandi, R. De Oliveira and L. 
Mastrostefano of the CERN advanced printed circuit workshop. This work would not have been possible without their skill and experience in the application of advanced $\mathrm{PCB}$ techniques.

\section{References}

[1] A Oed, et al, Nucl. Instr. and Meth. A263 (1988) 351.

[2] F Angelini, et al Nucl. Instr. and Meth. A283 (1989) 755.

[3] F Angelini, et al Nucl. Instr. and Meth. A355 (1993) 69.

[4] F Sauli, Nucl. Instr. and Meth. A386 (1997) 531.

[5] R Bellazzini, et al, The Micro-Groove Detector, to be published in NIM.

[6] A Gandi, et al, Manufacturing procedures for the Gas Electron Multiplier, in preparation at CERN (1997).

[7] R Bellazzini, et al, What is the real gas gain of a standard GEM?, Proceedings of the 8th Vienna Wire Chamber Conference (1998), to be published in NIM.

[8] J Benlloch, et al, Further developments and beam tests of the Gas Electron Multiplier (GEM), Proceedings of the 8th Vienna Wire Chamber Conference (1998), to be published in NIM.

[9] B Schmidt, Microstrip Gas Chambers: Recent developments, radiation damage and long term behaviour, Proceedings of the 8th Vienna Wire Chamber Conference (1998), to be published in NIM.

[10] P K Mallick, Fibre-reinforced composites materials, manufacturing and design, 2nd ed., Marcel Dekker, Inc., New York (1993).

[11] R Bellazzini and M A Spezziga, Electric field, avalanche growth and signal development in micro-strip gas chambers and micro-gap chambers, La Rivista del Nuovo Cimento della Societa Italiana di Fisica, Vol. 17, No. 12 (1994).

[12] R Bellazzini, et al, Technique for the characterisation of discharges in micro-strip gas chambers, Nucl. Instr. and Meth. A398 (1997) 426.

[13] CMS Technical Design Report, CERN/LHCC 98-6 (1998). 